7. Petrova, T.V., et al. 2002. Lymphatic endothelial reprogramming of vascular endothelial cells by the Prox-1 homeobox transcription factor. EMBO J. 21:4593-4599.

8. Kriehuber, E., et al. 2001. Isolation and characterization of dermal lymphatic and blood endothelial cells reveal stable and functionally specialized cell lineages. J. Exp. Med. 194:797-808

9. Makinen, T., et al. 2001. Isolated lymphatic endothelial cells transduce growth, survival and migratory signals via the VEGF-C/D receptor VEGFR-3. EMBO J. 20:4762-4773.

10. Maruyama, K., et al. 2005. Inflammation-induced lymphangiogenesis in the cornea arises from CD $11 \mathrm{~b}$ positive macrophages. J. Clin. Invest. 115:2363-2372. doi:10.1172/JCI23874.

11. Peters, B.A., et al. 2005. Contribution of bone marrow-derived endothelial cells to human tumor vasculature. Nat. Med. 11:261-262.

12. Mandriota, S.J., et al. 2001. Vascular endothelial growth factor-C-mediated lymphangiogenesis promotes tumour metastasis. EMBOJ. 20:672-682.

13. Skobe, M., et al. 2001. Induction of tumor lymph- angiogenesis by VEGF-C promotes breast cancer metastasis. Nat. Med. 7:192-198.

14. Schoppmann, S.F., et al. 2002. Tumor-associated macrophages express lymphatic endothelial growth factors and are related to peritumoral lymphangiogenesis. Am. J. Pathol. 161:947-956.

15. Cursiefen, C., Chen, L., Dana, M.R., and Streilein, J.W. 2003. Corneal lymphangiogenesis. Cornea. 22:273-281.

16. He, Y., et al. 2004. Preexisting lymphatic endothelium but not endothelial progenitor cells are essential for tumor lymphangiogenesis and lymphatic metastasis. Cancer Res. 64:3737-3740.

17. Salven, P., Mustjoki, S., Alitalo, R., Alitalo, K., and Rafii, S. 2003. VEGFR-3 and CD133 identify a population of CD34+ lymphatic/vascular endothelial precursor cells. Blood. 101:168-172.

18. Cursiefen, C., et al. 2004. VEGF-A stimulates lymphangiogenesis and hemangiogenesis in inflammatory neovascularization via macrophage recruitment. J. Clin. Invest. 113:1040-1050. doi:10.1172/JCI200420465.

19. Kerjaschki, D., et al. 2004. Lymphatic neoangiogen- esis in human kidney transplants is associated with immunologically active lymphocytic infiltrates. J. Am. Soc. Nephrol. 15:603-612.

20. Skobe, M., et al. 2001. Concurrent induction of lymphangiogenesis, angiogenesis, and macrophage recruitment by vascular endothelial growth factor-C in melanoma. Am. J. Pathol. 159:893-903.

21. Baluk, P., et al. 2005. Pathogenesis of persistent lymphatic vessel hyperplasia in chronic airway inflammation. J. Clin. Invest. 115:247-257. doi:10.1172/JCI200522037.

22. Tenstad, O., Heyeraas, K.J., Wiig, H., and Aukland, K. 2001. Drainage of plasma proteins from the renal medullary interstitium in rats. J. Physiol. 536:533-539.

23. Podgrabinska, S., et al. 2002. Molecular characterization of lymphatic endothelial cells. Proc. Natl. Acad. Sci. U. S. A. 99:16069-16074.

24. Hirakawa, S., et al. 2005. VEGF-A induces tumor and sentinel lymph node lymphangiogenesis and promotes lymphatic metastasis. J. Exp. Med. 201:1089-1099.

\title{
Searching for transcriptional regulators of Ang II-induced vascular pathology
}

\author{
Victor J. Dzau' and Marco Lopez-llasaca²
}

1Department of Medicine, Duke University Medical Center, Durham, North Carolina, USA. ${ }^{2}$ Department of Medicine, Brigham and Women's Hospital, Harvard Medical School, Boston, Massachusetts, USA.

\begin{abstract}
Ang II plays a key role in cardiovascular regulation and participates in vascular pathobiology, including inflammation and remodeling. Whether these tissue effects are mediated by direct Ang II actions or indirectly as a result of its influence on hemodynamics is being debated. In vitro data have shown that Ang II induces vascular cellular transcriptional activation and gene expression, but the mechanisms explaining its long-term tissue effects in vivo are relatively unknown. Do the multiple in vivo vascular activities elicited by Ang II (such as inflammation, fibrosis, and vascular cell hypertrophy/proliferation) occur via independent pathways, or do common transcription mechanisms mediate these multiple effects? In this issue, Zhan et al. identify Ets-1 as a critical downstream transcriptional mediator of vascular inflammation and remodeling in vivo; their data suggest that Ets-1 may be a common denominator of a complex process that involves multiple pathways previously considered to be mechanistically independent (see the related article beginning on page 2508). Characterization of the critical transcription programs activated by Ang II in vivo and determination of the hierarchy of responses are vital to the understanding of the mechanism of vascular disease and to the development of therapies targeted at inhibiting the common transcription effectors of vascular pathology.
\end{abstract}

Inflammation and structural remodeling are essential processes mediating vascular responses to humoral and hemodynamic

Nonstandard abbreviations used: KLF5, Krüppel-like factor 5; RAS, renin-angiotensin system; SRF, serum response factor.

Conflict of interest: The authors have declared that no conflict of interest exists.

Citation for this article: J. Clin. Invest. 115:2319-2322 (2005). doi:10.1172/JCI26384. stimuli and have been shown to be involved in the mechanism of vascular disease. Over the past several years, experimental and clinical evidence has demonstrated a key role of Ang II in these processes and that blockade of the renin-angiotensin system (RAS) inhibits vascular inflammation and remodeling and reduces clinical vascular complications. However, an ongoing debate exists with respect to the cardio- vascular actions of Ang II and prompts the following questions: (a) Does Ang II have direct in vivo vascular actions independent of its effects on blood pressure? (b) How does Ang II exert its long-term effects in tissue? and (c) Are the multiple Ang II-induced vascular pathologic processes (i.e., inflammation, fibrosis, and proliferation) mediated by common downstream transcription factor(s)?

Certainly, it has been difficult to dissect, in either in vivo or in vitro studies, the direct effect of Ang II on tissue from the indirect effects resulting from its influence on hemodynamics. Clarifying how the effects of Ang II are mediated will be of great clinical relevance, particularly with respect to the question of whether drugs that block RAS (e.g., angiotensinconverting enzyme inhibitors and Ang II receptor blockers) will have any added value beyond their traditional role as blood pressure-lowering agents. Although many in vitro studies have shown that Ang II activates signal transduction pathways, transcriptional activation, and gene expression, these studies have focused on acute experimental conditions, and data explaining the long-term cardiovascular actions of Ang II are lacking. The mecha- 


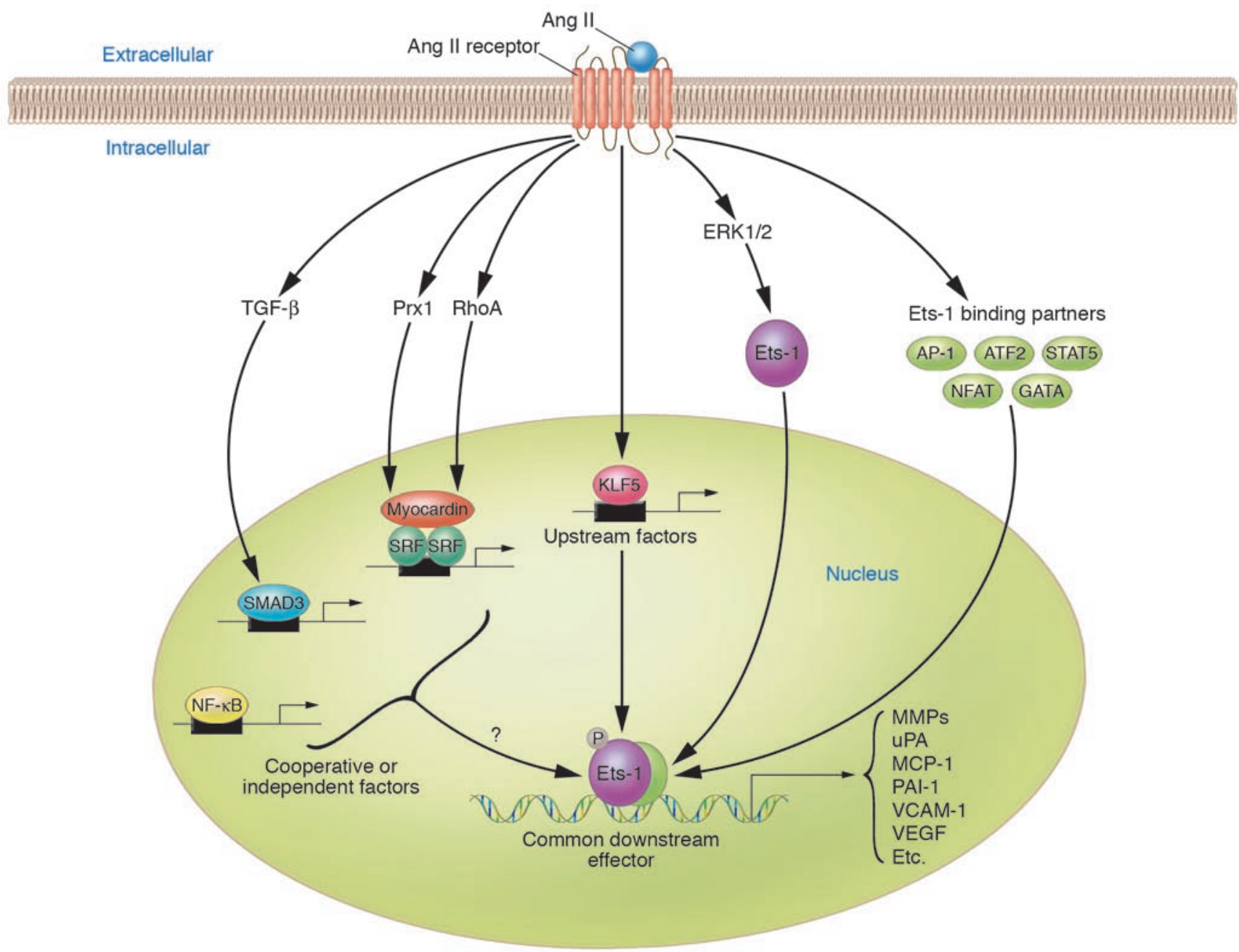

Figure 1

Mechanisms of Ang II-dependent activation of transcriptional programs that lead to changes in vascular function. Ang II activates several groups of transcriptional mechanisms that act in cooperation with Ets-1: (a) Ets-1 binding partners such as activator protein 1 (AP-1), STAT5, nuclear factor of activated T cells (NFAT), GATA, or activating transcription factor 2 (ATF2), which are able to physically bind and regulate Ets-1 activity; (b) KLF5, which is activated in a position upstream of Ets-1; and (c) factors that have been shown to influence vascular function in an Ets-1-independent or -cooperative fashion. The sequence of events that leads to the long-term changes in Ets-1 activation and vascular function in response to Ang II is still undefined. MCP-1, monocyte chemoattractant protein-1; PAI-1, plasminogen activator inhibitor-1; uPA, urokinase type plasminogen activator.

nisms responsible for Ang II activity, from acute to chronic, especially with respect to its effects on transcription regulation, are relatively unknown.

In this context, the study by Zhan et al. in this issue of the JCI (1) provides insight into an important downstream transcription mechanism mediating vascular inflammation and remodeling in response to chronic Ang II infusion. The authors demonstrate that Ets-1 is a critical mediator of vascular remodeling and inflammation in response to Ang II. They show that vascular hyperplasia, perivascular fibrosis, and cardiac hypertrophy are significantly diminished in Ets $1^{-/}$ mice compared with control mice in response to systemic administration of Ang II. Importantly, these responses occurred independently of any alterations in blood pressure. Furthermore, the induction of 2 known targets of Ets-1, cyclin-dependent kinase inhibitor p21 and plasminogen activator inhibitor-1, by Ang II was markedly blunted in the aorta of Ets $1^{-/-}$mice compared with wild-type controls. Monocyte chemoattractant protein-1, which had not been previously linked to Ets-1 transcriptional activation, was identified as a novel target for Ets-1. Interestingly, the authors also reported a significant reduction in recruitment of $\mathrm{T}$ cells and macrophages to the vessel wall, which supports a critical role for Ets-1 as a transcriptional mediator of vascular inflammation and remodeling in response to Ang II.

\section{Programs of transcriptional activation in response to Ang II}

Ets- 1 is the founder member of the Ets family of transcription factors that specifically recognizes and activates DNA response sequences that contain a GGAA/T core in the promoters of target genes (2). The DNA-binding activity of Ets-1 is controlled by phosphorylation and association with other transcription factors. Phosphorylation by ERK1/2 activates Ets-1, whereas phosphorylation by $\mathrm{Ca}^{2+}$ - 
calmodulin kinase II or myosin light chain kinase inhibits Ets-1 DNA-binding activity. A number of transcription factors have been shown to regulate the transcriptional activity of Ets- 1 by modulating Ets-1 DNA-binding affinity, such as acute myeloid leukemia-1, pituitary-specific transacting factor 1 , hypoxia-inducible factor $2 \alpha$, and $\operatorname{Pax} 5$ (3). In addition, the interaction of Ets-1 with GATA3, activating transcription factor $2, \mathrm{NF}-\kappa \mathrm{B}$, and Stat 5 cooperatively activates target promoters. Ets-1 increases the transcriptional activation of immediate-early gene promoters including a number of genes involved in VSMC growth and proliferation, endothelial activation, and the vascular inflammatory response. Ets-1 also induces the expression of MMP-1, -3 , and -9 and urokinase type plasminogen activator; these proteases are known to be involved in extracellular matrix degradation and atherosclerosis progression $(4,5)$. Ets- 1 activation therefore represents a final effector of multiple signaling pathways and components of protein complexes on immediate-early promoters. Indeed, the data reported by Zhan et al. (1) show that Ets- 1 is a downstream transcriptional mediator of Ang II-regulated expression of genes involved in the processes of vascular inflammation and remodeling.

Multiple signaling mechanisms potentially link Ang II to Ets-1-dependent transcription (Figure 1). Ang II activates the Ras-MAPK pathway, which in turn induces both the expression and phosphorylation of Ets-1, leading to expression of a number of genes involved in vascular responses. Besides the important role of Ets-1 as an effector of Ang II responses, several other mechanisms of transcriptional control involving nuclear factor of activated $\mathrm{T}$ cells, PI3K, AKT, RhoA, or myocardin, acting in concert with or independently of Ets-1, have begun to be characterized (6-8). For example, Ang II induces the binding of serum response factor (SRF) to the specific smooth and cardiac muscle coactivator myocardin; furthermore, the homeodomain protein Prx1 strongly promotes SRF binding to its specific response element after Ang II stimulation. Thus, what has emerged is a multiple-signal transduction system that defines expression of immediateearly genes (via SRF-Ets factors) or tissue-restricted genes (via SRF-myocardin), acting through a combinatorial mechanism of protein-protein interactions in response to Ang II. The observation by Zhan et al. (1) of a decrease in vascular remodeling and inflammation in Ets-1$\mathrm{KO}$ animals in response to Ang II implies that this transcription factor is a critical regulator of multiple pathways that are active in different tissues and, more importantly, identifies Ets- 1 as a common denominator of pathways previously considered mechanistically independent.

The fact that a network of transcription factors and effector proteins participate in the control of Ang II-dependent vascular remodeling and inflammation raises the question of how these proteins interact with each other and how they coordinate their activities to generate and maintain a specific vascular phenotype. Despite many in vitro observations linking Ang II to a multitude of effectors in VSMC growth and differentiation, there are few reports examining the specific role of these molecules with respect to the in vivo chronic effects of Ang II on the cardiovascular system. Studies in KO mice lacking expression of bradykinin receptor type 2, p66Shc, PI3K $\gamma$, or Krüppel-like factor 5 (KLF5) have shown that these effectors are critical for vascular responses to Ang II (9-11). However, the hierarchy of responses of these mediators and the place of action of transcription factors such as Ets-1 after the initial Ang II stimulus are unknown. The demonstration by Zhan et al. (1) that Ang II-dependent KLF5 activation occurs upstream of Ets-1 in vivo suggests that Ets-1 is a critical downstream regulator of a complex process that involves a multitude of effectors. The availability of specific KO animals will make it feasible to conduct epistasis analysis to establish the proper order of action of these important factors in Ang II-dependent vascular responses. Furthermore, given its critical role in transcriptional regulation, Ets-1 may be an important therapeutic target for the inhibition of vascular disease.

\section{Ang II-dependent gene expression: key unanswered questions}

Despite considerable progress over the past several years in defining the mechanisms of Ang II-dependent gene expression, significant gaps exist in our understanding of the means by which the cellular machinery controls the short- and long-term responses to Ang II at the transcriptional level. These responses include both the pathways activated by cellular receptors and activation of general intracellular sig- naling pathways following receptor activation. This raises the question of how the cellular machinery dictates the response from an acute signal pathway to longterm gene expression. In this regard, it has become evident in recent years that after the initial steps of ligand activation and desensitization, the Ang II receptor is still able to transduce intracellular signals due to the scaffold nature of $\beta$-arrestins bound to the receptor (12). Furthermore, after the initial stimulation, Ang II must induce genetic reprogramming in order to have a sustained effect on VSMC growth, which would therefore provide a link between the acute and chronic outcomes of Ang II actions. This reprogramming should set the subsequent level of local biochemical activities, even in the absence of receptor activation, which can have a long-term influence on the phenotype. The molecular nature of this genetic reprogramming and the determination of the existence of a master regulator are key to the understanding of chronic Ang II effects in tissues.

This knowledge will be critical to our understanding of the physiological and pathological roles of Ang II-dependent gene expression, which may then be applied in the treatment of human disease. The use of a comprehensive systems biology approach, including functional proteomics and high-throughput genomic techniques, to characterize the genetic program of vascular remodeling will help us better understand the complexity of Ang II-dependent gene expression in biological systems.

\section{Acknowledgments}

V.J. Dzau is supported by NIH grants HL058516, HL035610, HL072010, and HL073219. Marco Lopez-Ilasaca is supported by American Heart Association Scientist Development Grant 0435427T.

Address correspondence to: Victor J. Dzau, Office of the Chancellor, Duke University Medical Center, Durham, North Carolina 27708, USA. Phone: (919) 684-2255; Fax: (919) 681-7020; E-mail: victor.dzau@ duke.edu.

1. Zhan, Y., et al. 2005. Ets-1 is a critical regulator of Ang II-mediated vascular inflammation and remodeling. J. Clin. Invest. 115:2508-2516. doi:10.1172/JCI24403.

2. Dittmer, J. 2003. The biology of the Ets 1 protooncogene [review]. Mol. Cancer. 2:29.

3. Garvie, C.W., Pufall, M.A., Graves, B.J., and Wolberger, C. 2002. Structural analysis of the autoinhibition of Ets- 1 and its role in protein partnerships. J. Biol. Chem. 277:45529-45536. 
4. Baillat, D., Begue, A., Stehelin, D., and Aumer cier, M. 2002. ETS-1 transcription factor binds cooperatively to the palindromic head to head ETS-binding sites of the stromelysin- 1 promoter by counteracting autoinhibition. J. Biol. Chem. 277:29386-29398.

5. Iwasaka, C., Tanaka, K., Abe, M., and Sato, Y. 1996. Ets-1 regulates angiogenesis by inducing the expression of urokinase-type plasminogen activator and matrix metalloproteinase- 1 and the migration of vascular endothelial cells. J. Cell. Physiol. 169:522-531.

6. Dandre, F., and Owens, G.K. 2004. Platelet-derived growth factor-BB and Ets-1 transcription fac- tor negatively regulate transcription of multiple smooth muscle cell differentiation marker genes. Am. J. Physiol. 286:H2042-H2051.

7. Kuwahara, K., Barrientos, T., Pipes, G.C., Li, S., and Olson, E.N. 2005. Muscle-specific signaling mechanism that links actin dynamics to serum response factor. Mol. Cell. Biol. 25:3173-3181.

8. Lockman, K., et al. 2004. Sphingosine 1-phosphate stimulates smooth muscle cell differentiation and proliferation by activating separate serum response factor co-factors. J. Biol. Chem. 279:42422-42430.

9. Graiani, G., et al. 2005. Genetic deletion of the p66Shc adaptor protein protects from angiotensin II-induced myocardial damage. Hypertension.
46:433-440.

10. Vecchione, C., et al. 2005. Protection from angiotensin II-mediated vasculotoxic and hypertensive response in mice lacking PI3Kgamma. J. Exp. Med. 201:1217-1228.

11. Shindo, T., et al. 2002. Krüppel-like zinc-finger transcription factor KLF5/BTEB2 is a target for angiotensin II signaling and an essential regulator of cardiovascular remodeling. Nat. Med. 8:856-863.

12. Kohout, T.A., and Lefkowitz, R.J. 2003. Regulation of $\mathrm{G}$ protein-coupled receptor kinases and arrestins during receptor desensitization. Mol. Pharmacol. 63:9-18.

\title{
Osteoblast-derived PTHrP is a physiological regulator of bone formation
}

\author{
T. John Martin
}

St. Vincent's Institute of Medical Research and Department of Medicine, University of Melbourne, Melbourne, Victoria, Australia.

\begin{abstract}
Parathyroid hormone-related protein (PTHrP) acts as a paracrine regulator in several tissues, and its physiological roles also extend to bone. In this issue of the JCI, Miao et al. demonstrate that osteoblast-specific ablation of Pthrp in mice results in osteoporosis and impaired bone formation both in vivo and ex vivo (see the related article beginning on page 2402). These mice recapitulate the phenotype of mice with haploinsufficiency of Pthrp. The findings demonstrate that PTHrP plays a central role in the physiological regulation of bone formation, by promoting recruitment and survival of osteoblasts, and probably plays a role in the physiological regulation of bone resorption, by enhancing osteoclast formation. This has implications for both our understanding of the pathogenesis of osteoporosis and its treatment.
\end{abstract}

\section{Multiple sites of PTHrP production and action}

Parathyroid hormone-related protein (PTHrP) resembles parathyroid hormone (PTH) in its aminoterminal sequence (1), and the two have very similar structural requirements for binding and activation of their common receptor, type 1 PTH receptor (PTHR1) (2). Although PTHrP circulates as a hormone that causes hypercalcemia in patients with certain cancers, it has no normal hormonal role except in the fetus, where it promotes the transfer of calcium across the placenta from mother to fetus, and during late pregnancy and lactation, in which its function is still not fully defined $(3,4)$. Largely as a result of genetics-based studies, we now realize that PTHrP should not

Nonstandard abbreviations used: PTH, parathyroid hormone; PTHR1, type 1 PTH receptor; PTHrP, PTHrelated protein; RANK, receptor activator of NF-KB; RANKL, RANK ligand.

Conflict of interest: The author is a consultant to the Chugai Pharmaceutical Co.

Citation for this article: J. Clin. Invest. 115:2322-2324 (2005). doi:10.1172/JCI26239. be regarded as a hormone at all in its normal role. The physiological role of PTHrP in postnatal mammals appears to relate to its function as a paracrine effector. PTHrP protein and mRNA are both widely expressed in human tissues $(3,5)$, often playing important roles during both development and adulthood. Much of our understanding of the role of PTHrP has come from the analysis of genetically manipulated mice. For example, PTHrP has been shown to regulate epithelial-mesenchymal signaling during the development of mammary glands and hair follicles, as well as the relaxation of uterine and vascular smooth muscle (4). The fact that PTHrP mRNA is unstable, and that the protein itself is liable to extensive proteolytic processing, ideally equips $\mathrm{PTHrP}$ to function as a cytokine. The status of PTHrP as a paracrine or autocrine factor has been the subject of several reviews (3-6).

\section{PTHrP in bone}

The discovery of PTHrP production in bone $(7,8)$ pointed to possible local functions of this peptide, and ablation of either
Pthrp or Pthr1 in mice revealed a role for PTHrP in endochondral bone formation $(9,10)$. Targeted disruption of these genes in mice resulted in gross skeletal abnormalities consistent with chondrodysplasia, and death in the perinatal period $(9,10)$. PTHrP mRNA and protein were shown to be produced also in intramembranous bone, by cells at even the earliest mesenchymal stage of development (11). Other in vitro and in vivo observations indicated that PTHrP and PTHR1 were expressed by different cell populations. Cells of mesenchymal lineage that differentiate into osteoblasts were shown to produce PTHrP very early during differentiation, and PTHrP levels decreased with further cell maturation. PTHR1 is expressed at a later stage in differentiation, by committed preosteoblasts $(7,8)$.

\section{PTH, the hormone; PTHrP, the bone cytokine}

Some years ago Amizuka et al. showed that although thrp $^{-/-}$mice died at the time of birth because of a cartilage defect, Pthrp ${ }^{+/-}$mice survived. By 3 months of age the haplotype-insufficient mice were markedly osteoporotic and possessed an increased number of adipocytes in their bone marrow (9). These observations gave much impetus to the view that PTHrP is a local factor of some importance in bone. In order to determine the relative contributions to bone physiology of the endocrine factor PTH and the paracrine factor PTHrP, Miao et al. recently examined mice rendered null for $P$ th and found that they had increased trabecular bone volume because 